\title{
Suppression of anharmonic phonons and $s$-wave superconductivity by defects in the filled skutterudite $\mathrm{LaRu}_{4} \mathrm{As}_{12}$
}

\author{
Y. Mizukami $\odot,{ }^{1, *}$ M. Kończykowski $\odot,{ }^{2}$ O. Tanaka, ${ }^{1}$ J. Juraszek $\odot,{ }^{3}$ Z. Henkie $\odot,{ }^{3}$ T. Cichorek $\odot,{ }^{3}$ and T. Shibauchi $\odot^{1}$ \\ ${ }^{1}$ Department of Advanced Materials Science, University of Tokyo, Kashiwa, Chiba 277-8561, Japan \\ ${ }^{2}$ Laboratoire des Solides Irradiés, CEA/DRF/IRAMIS, Ecole Polytechnique, CNRS, Institut Polytechnique de Paris, \\ F-91128 Palaiseau, France \\ ${ }^{3}$ Institute of Low Temperature and Structure Research, Polish Academy of Sciences, 50-422 Wroctaw, Poland
}

(Received 7 September 2020; revised 26 October 2020; accepted 2 December 2020; published 28 December 2020)

\begin{abstract}
In filled-skutterudite compounds, guest atoms are centered at highly symmetric cages essentially formed by pnictogens. The weak bonding of the guest atoms to the cage-forming elements is expected to give rise to local atomic vibrations in anharmonic potential which can influence electronic properties and may even promote superconductivity. However, the relation between the unusual low-energy phonon excitations and the electronic properties is still elusive, and evidence establishing a direct link between them is a long-standing issue. Here, we investigate the impact of artificial atomic defects introduced by electron irradiation in the filled-skutterudite superconductor $\mathrm{LaRu}_{4} \mathrm{As}_{12}$. Our high-resolution heat capacity measurements reveal that the electronic specific heat is substantially reduced with increasing concentration of atomic defects. Moreover, the irradiation suppresses the fully gapped $s$-wave superconductivity as well as the contribution of anharmonic phonons in the specific heat in a correlated fashion. Our findings imply that the anharmonic phonons play an important role in enhancing electronic specific heat and superconductivity.
\end{abstract}

DOI: 10.1103/PhysRevResearch.2.043428

\section{INTRODUCTION}

In the cage compounds such as $\mathrm{Ge} / \mathrm{Si}$ clathrates, $\beta$ pyrochlore oxides, and filled skutterudites, the crystal structures are composed of large atomic cages which contain guest atoms at the center of the cages [1-6]. This cage structure often gives rise to the large atomic vibrations of the guest atoms in the anharmonic potential inside the cages. This characteristic atomic oscillation is sometimes called rattling and may induce unusual electronic properties such as high thermoelectronic efficiency $[2,7,8]$ or may promote superconductivity in some materials [9]. Recently, such anharmonicity of phonons has been studied also in the high-transition-temperature superconductivity in pressurized sulfur hydride systems [10-13].

The filled-skutterudite compounds $[5,6,14]$, where the lanthanoid atoms are surrounded by pnictogen cages, exhibit a variety of electronic states such as non-Fermi liquid behavior [15], exotic heavy-fermion properties [16,17], two-band and unconventional superconductivity [18-21], low-temperature ferromagnetic order [22-25], and multipole ordering [26,27]. In the filled skutterudites, anharmonic vibrations of lanthanoid atoms are often observed, and hence these materials offer a

\footnotetext{
*mizukami@edu.k.u-tokyo.ac.jp

Published by the American Physical Society under the terms of the Creative Commons Attribution 4.0 International license. Further distribution of this work must maintain attribution to the author(s) and the published article's title, journal citation, and DOI.
}

rare opportunity for experimental investigations of the theoretically predicted impact of the cage-structure-driven degrees of freedom on the electronic properties. Specifically, it remains unclear how anharmonic phonons affect the normal-state electron properties and the superconductivity.

In this regard, the filled-skutterudite superconductor $\mathrm{LaRu}_{4} \mathrm{As}_{12}$ [28] is a suitable system, where lanthanum does not contain $f$ electrons which may alter the electronic properties via the Kondo effect. $\mathrm{LaRu}_{4} \mathrm{As}_{12}$ exhibits relatively large Sommerfeld coefficient $\gamma \approx 60 \mathrm{~mJ} \mathrm{~mol}^{-1} \mathrm{~K}^{-2}[29,30]$, which deviates from the value of band structure calculation by roughly a factor of 2 [31-33]. Moreover, the superconductivity occurs at the superconducting transition temperature $T_{c}=10.4 \mathrm{~K}$, which is the highest among the stoichiometric filled-skutterudite compounds [28]. Therefore, the relation between anharmonic phonons and electron properties can solely be investigated without the contribution from $f$ electrons.

Here we have studied the impact of artificial atomic defects in crystal lattices on the normal-state and superconducting properties in single crystalline $\mathrm{LaRu}_{4} \mathrm{As}_{12}$. The atomic defects introduced by electron irradiation deform the cage structure and lower the symmetry of the potential. Through the heat capacity measurements on the samples with different amounts of atomic defects, we find that the Sommerfeld coefficient $\gamma$ is significantly reduced with introduction of defects. Moreover, the introduction of defects also reduces the anharmonic phonon contribution to the total heat capacity and suppresses the superconductivity. Our observation implies that the anharmonic vibrations of La ion play an important role in the enhancement of the Sommerfeld coefficient and/or superconductivity in this compound. 
(a)
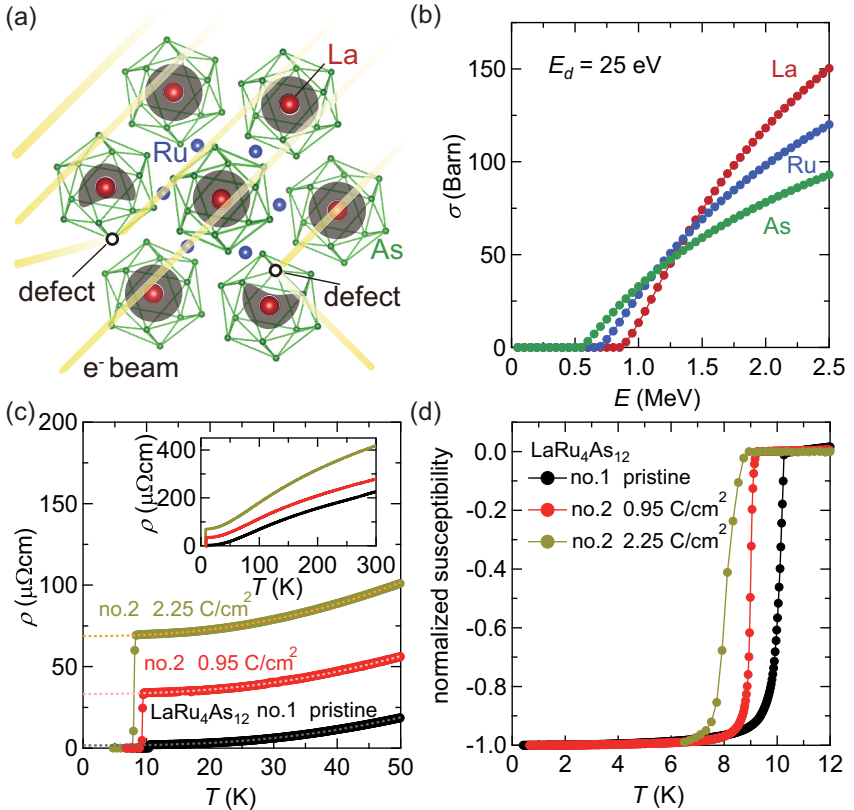

FIG. 1. Electron irradiation of $\mathrm{LaRu}_{4} \mathrm{As}_{12}$. (a) Schematic illustration of atomic defects introduced by electron irradiation on the cage structure of $\mathrm{LaRu}_{4} \mathrm{As}_{12}$. The crystal structure is viewed along the [111] crystal axis. The defects introduced by irradiation deform the cage and lower the symmetry of potential inside the cage which is illustrated as the grey area. (b) Cross section $\sigma$ as a function of incident electron energy $E$ for $\mathrm{La}, \mathrm{Ru}$, and As ions, respectively. $E_{d}$ is the displacement energy for the creation of atomic defects used in this calculation. The cross section does not have strong dependence on $E_{d}$. (c) $\rho(T)$ at low temperatures for $\mathrm{LaRu}_{4} \mathrm{As}_{12}$ samples with different dose levels. The dashed line represents the fitting result to power-law temperature dependence. The inset shows $\rho(T)$ in a wider temperature range. (d) $T$ dependence of magnetic susceptibility normalized at the lowest $T$ for $\mathrm{LaRu}_{4} \mathrm{As}_{12}$ samples with different dose levels.

\section{ELECTRON IRRADIATION}

The atomic defects are artificially introduced by electron irradiation in a controllable manner, where the incident electron energy is tuned at $2.5 \mathrm{MeV}$ to create uniformly distributed atomic-scale defects [34,35]. The schematic picture of the electron irradiation is illustrated in Fig. 1(a). The amount of defects in the crystals is proportional to the irradiation dose $\left(\mathrm{C} / \mathrm{cm}^{2}\right)$ through the cross section of each element contained in the compound. Figure 1(b) depicts the cross section as a function of incident electron energy for $\mathrm{La}, \mathrm{Ru}$, and As atoms, showing that the cross section for La is the largest at $2.5 \mathrm{MeV}$. However, there are $12 \mathrm{As}$ atoms in the unit cell of $\mathrm{LaRu}_{4} \mathrm{As}_{12}$, and thus As atoms are mainly removed by irradiation. This results in the deformation of the cage structure and the lowering of the cage symmetry. Using the value of the cross section, the displacement per atom can be roughly estimated to be close to one percent per unit cell for $1 \mathrm{C} / \mathrm{cm}^{2}$ irradiation dose. The introduced atomic defects can be confirmed by the temperature dependence of resistivity $\rho(T)$, measured by the standard four-probe method. Figure 1(c) shows the resistivity for $\mathrm{LaRu}_{4} \mathrm{As}_{12}$ samples with different dose levels. At low temperatures, the $\rho(T)$ curves in the normal state can be fitted with power law $\rho(T)=\rho_{0}+A T^{\alpha}$ with the exponent
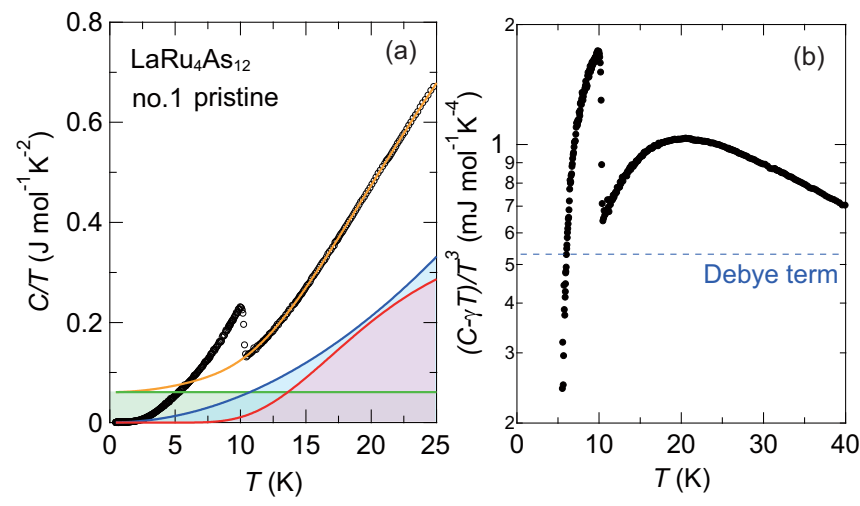

FIG. 2. Analysis of specific heat of $\mathrm{LaRu}_{4} \mathrm{As}_{12}$ including the anharmonic phonon term described by Einstein model. (a) Temperature dependence of $C(T)$ divided by $T$. Black open circles represent the raw data and the orange line is the fitting result above the superconducting transition temperature $T_{c}$. The green, blue, and red areas correspond to the contribution of electronic density of states, Debye phonons, and anharmonic phonons, respectively. (b) The phonon contributions $C(T)-\gamma T$ divided by $T^{3}$ as a function of $T$. The blue dashed line is the Debye term behaving as constant in this plot.

$\alpha \approx 2.5$, which is shown by the dashed lines in Fig. 1(c). It is clearly seen that the $T$-independent residual resistivity $\rho_{0}$ is drastically enhanced with increasing irradiation dose, indicating that nonmagnetic impurity scattering increases with irradiation. This fact verifies that the atomic defects are systematically introduced into the crystals by electron irradiation. It should be noted that at high temperatures $T \gtrsim 100 \mathrm{~K}, \rho(T)$ exhibits concave curvature for all samples as depicted in the inset of Fig. 1(c). This behavior implies that the inelastic scattering by anharmonic phonons is significant in $\mathrm{LaRu}_{4} \mathrm{As}_{12}$; theoretical calculations for a rattling system [36] reveal that $\rho(T)$ shows $\sqrt{T}$ dependence at higher temperatures than the characteristic energy scale of the anharmonic oscillation, and its amplitude depends on the amount of anharmonicity, which will be discussed later. In Fig. 1(d), the $T$ dependence of magnetic susceptibility measured by a commercial superconducting quantum interference device magnetometer at $10 \mathrm{Oe}$ normalized at the lowest temperatures is displayed for samples with different irradiation dose levels. Each sample shows the superconducting transition with similar sharpness as the one in pristine sample, which demonstrates that the introduced atomic defects are uniformly distributed inside the crystals.

\section{HEAT CAPACITY}

Having established that the uniformly distributed atomic defects are introduced inside the samples in a systematic manner, we next turn to the $T$ dependence of specific heat capacity $C(T)$ to see the change of the electronic properties with irradiation. Here we used the long relaxation method with a homemade cell designed for heat capacity measurements of small single crystals with mass of $\approx 10-100 \mu \mathrm{g}$. The solid black circles in Fig. 2(a) represent the $T$ dependence of $C(T) / T$ for pristine samples. The temperature dependence of $C(T) / T$ exhibits a sharp superconducting jump at $T_{c}=$ 10.4 K, consistent with the previous report, indicating high quality of the sample [29,33]. The specific heat above $T_{c}$ can 
usually be described by the combination of the electronic contribution term $\gamma T$ and Debye phonon term obeying $T^{3}$ dependence. In filled-skutterudite compounds, however, it is well known that the specific heat has a contribution from anharmonic phonons. Considering this fact, $C(T) / T$ can be given as $[9,30,37]$

$$
C(T) / T=\gamma+(1-x) N \beta T^{2}+x \frac{3 N R}{T}\left(\frac{T_{E} / 2 T}{\sinh \left(T_{E} / 2 T\right)}\right)^{2},
$$

where $\beta=12 \pi^{4} R /\left(5 T_{D}^{3}\right)$ in the second term is the coefficient of the Debye term, and the third term is the Einstein term describing the anharmonic phonon. Here, $T_{D}, T_{E}, x, N=17$, and $R$ are Debye temperature, Einstein temperature, relative weight of the Einstein term against Debye term, the number of oscillators in the unit cell, and molar gas constant, respectively. It should be noted that the Einstein model is indeed harmonic approximation of local atomic vibration, but it is well established that it describes the anharmonic mode in cage compounds [4]. The fitting is performed with the parameters $\gamma, T_{D}, T_{E}$, and $x$, and the result well reproduces the $C(T) / T$ data above $T_{C}$ as shown in Fig. 2(a) with the parameters $\gamma=60.8 \mathrm{~mJ} \mathrm{~mol}^{-1} \mathrm{~K}^{-2}, T_{D}=388.9 \mathrm{~K}, T_{E}=99.8 \mathrm{~K}$, and $x=0.055$, which are in good agreement with the previous analysis [30].

The anharmonic term can also be seen clearly when we plot the phonon heat capacity $[C(T)-\gamma T]$ divided by $T^{3}$ as a function of $T$ as shown in Fig. 2(b). The existence of the broad maximum above $T_{c}$ indicates that the phonon specific heat cannot be described only by Debye term. Although ultrasonic measurements do not clearly detect the dispersion originating from the anharmonic phonons [38], the Einstein term should be included in order to describe the total phonon specific heat, which has been discussed as an indication of the rattling guest ion modes in La-based filled skutterudites [30,39]. Here, the broad maximum appears at $\simeq T_{E} / 4.92$ in the Einstein model. From the peak temperature $\simeq 20.2 \mathrm{~K}$, the $T_{E}$ can be estimated to be $\simeq 99.4 \mathrm{~K}$, which is in good agreement with the fitting result in Fig. 2(a).

Next, we discuss the evolution of $C(T)$ with increasing atomic defect density. The solid circles in Figs. 3(a)-3(c) represent the $C(T) / T$ data for $1.02,2.25$, and $14.1 \mathrm{C} / \mathrm{cm}^{2}$ samples, which show superconducting jumps at $T_{c}=9.7,8.3$, and $5.5 \mathrm{~K}$, respectively. The fitting results by Eq. (1) are also shown as the solid lines and well reproduce the $C(T) / T$ data above $T_{c}$ for all samples. Figure 3(d) shows the $T$ dependence of the electronic contribution $C_{e}(T) / T$ for all samples, which is obtained by subtracting the two types of phonon contributions, the Debye and Einstein terms expressed in Eq. (1), from the total $C(T) / T$. The samples irradiated to the dose of 1.02 and $2.25 \mathrm{C} / \mathrm{cm}^{2}$ show clear jumps at $T_{c}$ with identical sharpness to the one in pristine sample, indicating that the two irradiated samples are as homogeneous as the pristine sample. Owing to the high level of the irradiation dose, the sample with $14.1 \mathrm{C} / \mathrm{cm}^{2}$ exhibits a broadened jump at $T_{c}=5.5 \mathrm{~K}$ compared to the samples with lower dose. However, the jump is quite discernible and the height of the jump is substantial against the normal-state electronic heat capacity $\gamma T$ above $T_{c}$, indicating that the bulk nature can safely be discussed.
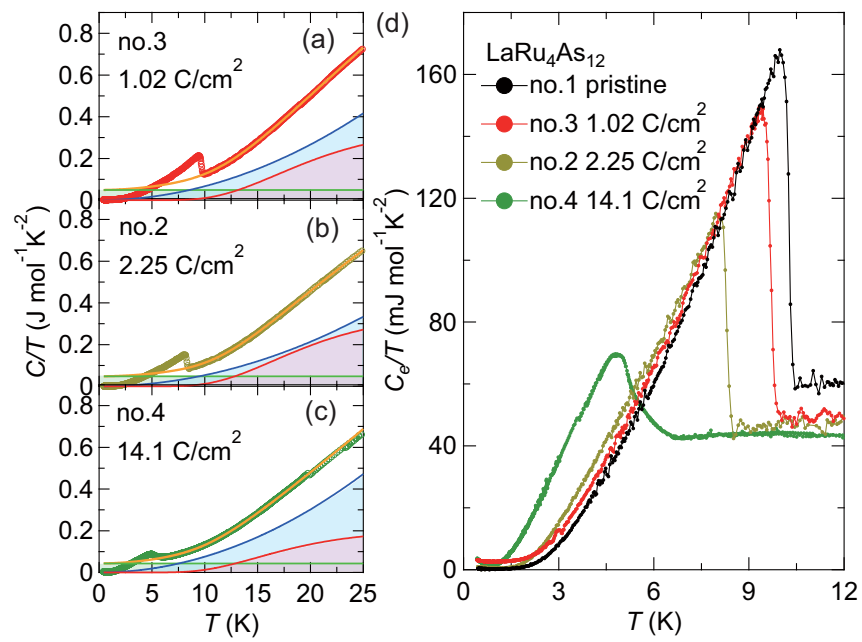

FIG. 3. Evolution of specific heat with electron irradiation. [(a)(c)] Solid circles represent $C(T) / T$ for the samples with 1.02 (a), 2.25 (b), and $14.1 \mathrm{C} / \mathrm{cm}^{2}$ (c). The solid orange lines represent the fitting results for all samples. Each component in the total specific heat is represented by colored area in the same way as Fig. 2(a). (d) $C_{e}(T) / T$ for the pristine sample and the samples irradiated to the dose of $1.02,2.25$, and $14.1 \mathrm{C} / \mathrm{cm}^{2}$ are represented by solid black circles, solid red circles, solid yellow circles, and solid green circles, respectively.

\section{DISCUSSION AND CONCLUSION}

The $C_{e}(T) / T$ data below $T_{c}$ for pristine sample exhibit a sharp decay at low temperatures and becomes almost flat below $3 \mathrm{~K}$. This $T$ dependence is consistent with the thermally activated BCS-type excitations, although it is reported that there are multiple gaps with different magnitudes [29,40,41]. This thermally activated behavior is quite robust against of $\mathrm{LaRu}_{4} \mathrm{As}_{12}$ irradiation, evidenced by the sharp decay also observed for all samples at low temperatures. Here, the atomic defects introduced by irradiation act as impurity scatterer for electrons. This fact indicates that there is no additional excitations of the Andreev bound states induced by the impurity scattering [35], showing unambiguously that there is no sign reversal in the superconducting gap as expected for $s$-wave fully gapped superconductors.

Figure 4(a) displays the change in $T_{c}$ with increasing irradiation dose determined by the heat capacity measurements, showing that $T_{c}$ almost linearly decreases with irradiation dose. Although the conventional $s$-wave superconductivity is expected to be robust against nonmagnetic impurity scattering known as Anderson's theorem, the irradiation substantially suppresses the superconductivity in $\mathrm{LaRu}_{4} \mathrm{As}_{12}$. This is indeed incompatible with the absence of the Andreev bound states, whose appearance is usually concomitant with the depression of $T_{c}$ in terms of the pair breaking in superconductors. One explanation for $T_{c}$ depression without Andreev bound states is the mixing of the gap between the different bands by scattering. Indeed, it is well known that the $T_{c}$ in multigap superconductor $\mathrm{MgB}_{2}$ is reduced with increasing impurity. This behavior is explained by averaging of the gap magnitude between the two bands, and the crossover occurs from two-gap to single-gap superconductivity with increasing impurity scattering [42]. 

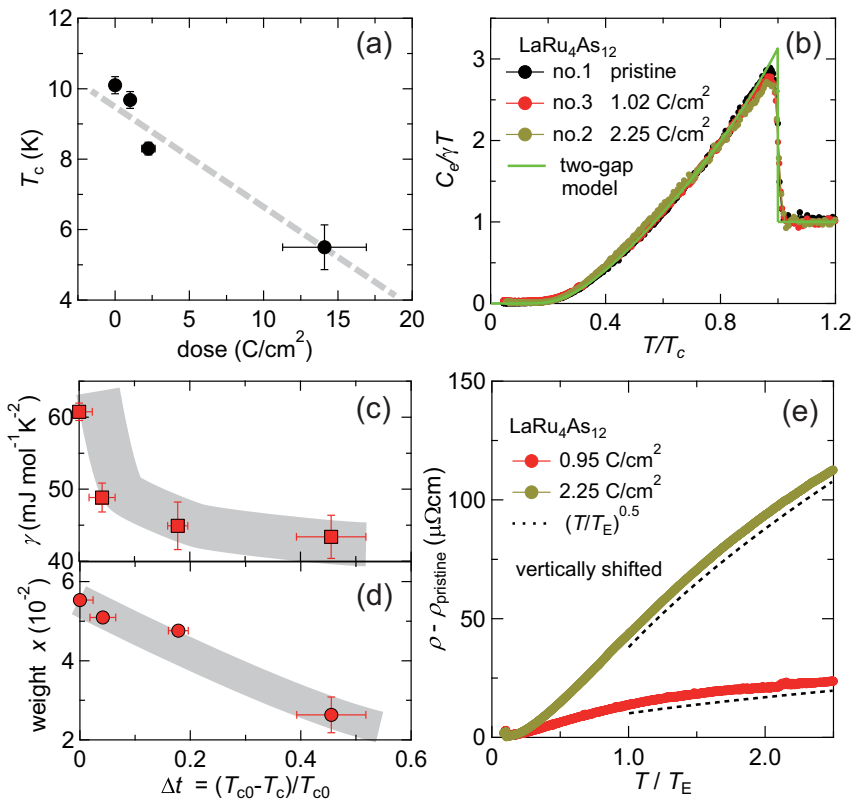

FIG. 4. Correlations between superconductivity, density of states, and anharmonic phonon weight in irradiated $\mathrm{LaRu}_{4} \mathrm{As}_{12}$. (a) The change in $T_{c}$ with irradiation dose. The dashed gray line is the guide for the eye. (b) $C_{e} / \gamma T$ for the pristine sample, and the samples irradiated to the dose of 1.02 and $2.25 \mathrm{C} / \mathrm{cm}^{2}$ are represented by solid black circles, solid red circles, and solid yellow circles, respectively. The solid green line represents the result of multigap analysis using one gap $\Delta_{1} / k_{B} T_{c}=2.7$ with the ratio of $35 \%$ and the other gap $\Delta_{2} / k_{B} T_{c}=1.8$ with the ratio of $65 \%$. (c) Sommerfeld coefficient $\gamma$ as a function of the relative reduction of $T_{c}$ normalized by the pristine $T_{c 0}, \Delta t=\left(T_{c 0}-T_{c}\right) / T_{c 0}$. The gray shade is the guide for the eye. (d) The change in the relative weight of anharmonic phonon against conventional Debye phonon with $\Delta t$. The gray shade is the guide for the eye. (e) Resistivity for irradiated samples plotted against $T / T_{E}$, where the pristine sample's resistivity $\rho_{\text {pristine }}$ is subtracted. The data are vertically shifted for clarity.

In order to check whether the averaging of the gap occurs in our case, we perform multigap analysis with two different superconducting gaps. Figure 4(b) depicts $C_{e}(T) / T$ normalized by $\gamma$ for pristine and 1.02 and $2.25 \mathrm{C} / \mathrm{cm}^{2}$ samples together with the result of the multigap analysis. Interestingly, the $C_{e}(T) / T$ curvatures for all samples including the pristine one roughly fall into a single curve, which is well reproduced by the result of multigap analysis with a single set of parameters. Here, the model employs the magnitude of one gap $\Delta_{1} / k_{B} T_{c}=2.7$ with the ratio of $35 \%$ and the other gap $\Delta_{2} / k_{B} T_{c}=1.8$ with the ratio of $65 \%$, which are in agreement with the previous multigap analysis [29]. Although a small feature is visible at around $0.5 T_{c}$ for the sample with $2.25 \mathrm{C} / \mathrm{cm}^{2}$, possibly due to the small change in the gap magnitude, this fact suggests that the effect of gap averaging is weak in this level of irradiation for $\mathrm{LaRu}_{4} \mathrm{As}_{12}$ and it is not the main origin for the $T_{c}$ depression.

Here, we point out that the irradiation reduces the $\gamma$ coefficient, which can be clearly seen as the decrease in the constant term of $C_{e}(T) / T$ above $T_{c}$ in Fig. 3(d). The $\gamma$ coefficient reflects the electronic density of states at Fermi energy $N\left(E_{F}\right)$, and it is quite natural that the reduction of $T_{c}$ correlates with the decrease of $\gamma$ in the conventional theoretical framework of superconductivity. Figure 4(c) displays the change of $\gamma$ with the reduction of $T_{c}$, showing that the $\gamma$ is suppressed with irradiation and tends to saturate. Then, the questions are why $\gamma$ is suppressed with increasing atomic defects in $\mathrm{LaRu}_{4} \mathrm{As}_{12}$ and why in the first place experimentally determined $\gamma \simeq 60$ $\mathrm{mJ} \mathrm{mol}{ }^{-1} \mathrm{~K}^{-2}$ is significantly larger than expected from band structure calculation [33]. One explanation for this is that the density of state may be highly sensitive to the dislocation of the As atoms. According to the band structure calculation, the density of states shows a peak structure near $E_{F}$, where the contribution from the As $4 p$ orbital is dominant [32,33]. However, it is not clear whether a small amount of defects can change the band structure properties significantly, and even if the peak structure is taken into account, it cannot be explained why the experimentally determined $\gamma$ is much larger than calculated $\gamma$. Another explanation is that the $d$ electron correlation effect may enhance the $\gamma$ coefficient, but it is not clear either whether the correlation effect exhibits such sensitivity to a small amount of defects as shown in the rapid decrease of $\gamma$ with irradiation. Here, it is theoretically proposed that the anharmonicity can enhance renormalization effect [43], and the large $\gamma$ values are indeed observed in some cage compounds $[4,44]$. Then, another possible explanation is that the anharmonic phonons enhance $\gamma$ also in $\mathrm{LaRu}_{4} \mathrm{As}_{12}$ and the cage deformation by atomic defects suppresses the anharmonic phonons, leading to the reduction of $\gamma$. Figure $4(d)$ shows the change in the weight of the anharmonic phonon contribution in the specific heat [see Eq. (1)] with decreasing $T_{c}$. The weight is decreased with increasing atomic defects and almost linearly correlates with the reduction of $T_{c}$. The suppression of the anharmonic phonons is also supported by the evolution of $\rho(T)$ with irradiation. It is theoretically suggested that $\rho(T)$ exhibits $\sqrt{T}$ dependence with concave curvature in the presence of the anharmonic phonons [36], and $\rho(T)$ for $\mathrm{LaRu}_{4} \mathrm{As}_{12}$ implies such a behavior as shown in the inset of Fig. 1(c). Figure 4(e) displays the change in $\rho(T)$ for irradiated samples as a function of $T / T_{E}$, where the resistivity data $\rho_{\text {pristine }}(T)$ for pristine sample is subtracted to extract the evolution of scattering by anharmonic phonons. It can be seen that the $T$ dependence above the characteristic energy scale of anharmonic phonons $T_{E}$ can be approximated by $\sqrt{T}$ dependence, but the concaveness is weaker in more irradiated sample with smaller weight of anharmonic phonons. This behavior is in good qualitative agreement with the theoretical calculation [36]. These results suggest that the anharmonic phonons enhance $\gamma$ coefficient in pristine $\mathrm{LaRu}_{4} \mathrm{As}_{12}$ and they are reduced with the introduction of atomic defects through the deformation of the cage structure. Because the anharmonic phonons originates from the flat potential, which is produced by the high cage symmetry, the displacement of only one of twelve As atoms can generate local minima of potential inside the cage. This mechanism thus can explain the substantial change in the anharmonic phonons by the small amount of defects. Indeed, the saturation of $\gamma$ with decreasing $T_{c}$ can be understood as approaching the band calculation value $32 \mathrm{~mJ} \mathrm{~mol}^{-1} \mathrm{~K}^{-2}$ [33] in the high dose regime, where there is less impact of anharmonic phonon on $\gamma$ coefficient. 
Several theoretical studies have also proposed that the anharmonic phonon itself can be a pairing glue for the superconductivity $[43,45]$. It is not clear whether the reduction of $T_{c}$ in $\mathrm{LaRu}_{4} \mathrm{As}_{12}$ with irradiation is a direct consequence of the suppression of anharmonic phonon or the secondary effect through the reduction of the $\gamma$ coefficient. In each case, our findings imply that the anharmonic phonons play an important role in enhancing electronic specific heat and/or superconductivity. This observation is achieved through the systematic introduction of artificial atomic defects in a given compound and demonstrates that the local structure such as atomic defects can be a tuning parameter which substantially changes the electronic specific heat and superconducting properties in cage compounds.

\section{ACKNOWLEDGMENTS}

We thank P. Chandra, P. Coleman, and Y. Nomura for discussion. Irradiation realized on the SIRIUS platform was supported by French national network of accelerators for irradiation and analysis of molecules and materials EMIR\&A under Projects No. 18-8161, No. 18-9931 and No. 202881. Growth of $\mathrm{LaRu}_{4} \mathrm{As}_{12}$ single crystals was supported by the Polish National Science Centre Project No. 2011/01/B/ST3/05685. This work was supported by Grants-in-Aid for Scientific Research (KAKENHI) (No. JP19H00649, No. JP20H02600, and No. JP20K21139) and Grant-in-Aid for Scientific Research on innovative areas "Quantum Liquid Crystals" (No. JP19H05824) from Japan Society for the Promotion of Science (JSPS).
[1] J. S. Kasper, P. Hagenmuller, M. Pouchard, and C. Cros, Clathrate structure of silicon $\mathrm{Na}_{8} \mathrm{Si}_{46}$ and $\mathrm{Na}_{x} \mathrm{Si}_{136}(x<11)$, Science 150, 1713 (1965).

[2] G. S. Nolas, J. L. Cohn, G. A. Slack, and S. B. Schujman, Semiconducting Ge clathrates: Promising candidates for thermoelectric applications, Appl. Phys. Lett. 73, 178 (1998).

[3] S. Yonezawa, Y. Muraoka, and Z. Hiroi, New $\beta$-pyrochlore oxide superconductor $\mathrm{CsOs}_{2} \mathrm{O}_{6}$, J. Phys. Soc. Jpn. 73, 1655 (2004).

[4] Z. Hiroi, J. Yamaura, and K. Hattori, Rattling good superconductor: $\beta$-pyrochlore oxides $\mathrm{AOs}_{2} \mathrm{O}_{6}$, J. Phys. Soc. Jpn. 81, 011012 (2012).

[5] W. Jeitschko and D. Braun, $\mathrm{LaFe}_{4} \mathrm{P}_{12}$ with filled $\mathrm{CoAs}_{3}$-type structure and isotypic lanthanoid-transition metal polyphosphides, Acta Crystallogr. Sec. B 33, 3401 (1977).

[6] H. Sato, H. Sugawara, Y. Aoki, and H. Harima, in Handbook of Magnetic Materials, edited by K. H. J. Buschow (NorthHolland, Amsterdam, 2009), Vol. 18, p. 1.

[7] B. C. Sales, D. Mandrus, and R. K. Williams, Filled skutterudite antimonides: A new class of thermoelectric materials, Science 272, 1325 (1996).

[8] X. Shi, J. Yang, J. R. Salvador, M. Chi, J. Y. Cho, H. Wang, S. Bai, J. Yang, W. Zhang, and L. Chen, Multiple-filled skutterudites: High thermoelectric figure of merit through separately optimizing electrical and thermal transports, J. Am. Chem. Soc. 133, 7837 (2011)

[9] Y. Nagao, J. Yamaura, H. Ogusu, Y. Okamoto, and Z. Hiroi, Rattling-induced superconductiviy in the $\beta$-pyrochlore oxides $\mathrm{AOs}_{2} \mathrm{O}_{6}$, J. Phys. Soc. Jpn. 78, 064702 (2009).

[10] I. Errea, M. Calandra, C. J. Pickard, J. Nelson, R. J. Needs, Y. Li, H. Liu, Y. Zhang, Y. Ma, and F. Mauri, High-Pressure Hydrogen Sulfide from First Principles: A Strongly Anharmonic Phonon-Mediated Superconductor, Phys. Rev. Lett. 114, 157004 (2015).

[11] D. A. Papaconstantopoulos, B. M. Klein, M. J. Mehl, and W. E. Pickett, Cubic $\mathrm{H}_{3} \mathrm{~S}$ around $200 \mathrm{GPa}$ : An atomic hydrogen superconductor stabilized by sulfur, Phys. Rev. B 91, 184511 (2015).

[12] I. Errea, F. Belli, L. Monacelli, A. Sanna, T. Koretsune, T. Tadano, R. Bianco, M. Calandra, R. Arita, F. Mauri, and J. A. Flores-Livas, Quantum crystal structure in the 250-kelvin superconducting lanthanum hydride, Nature (London) 578, 66 (2020).

[13] C. Setty, M. Baggioli, and A. Zaccone, Anharmonic phonon damping enhances the $T_{c}$ of BCS-type superconductors, Phys. Rev. B 102, 174506 (2020).

[14] M. B. Maple, N. A. Frederick, P.-C. Ho, W. M. Yuhasz, and T. Yanagisawa, Unconventional superconductivity and heavy fermion behavior in $\mathrm{PrOs}_{4} \mathrm{Sb}_{12}$, J. Supercond. Novel Magn. 19, 299 (2006).

[15] R. E. Baumbach, P.-C. Ho, T. A. Sayles, M. B. Maple, R. Wawryk, T. Cichorek, A. Pietraszko, and Z. Henkie, Non-Fermi liquid behavior in the filled skutterudite compound $\mathrm{CeRu}_{4} \mathrm{As}_{12}$, J. Phys.: Condens. Matter 20, 075110 (2008).

[16] S. Sanada, Y. Aoki, H. Aoki, A. Tsuchiya, D. Kikuchi, H. Sugawara, and H. Sato, Exotic heavy-fermion state in filled skutterudite $\mathrm{SmOs}_{4} \mathrm{Sb}_{12}$, J. Phys. Soc. Jpn. 74, 246 (2005).

[17] R. E. Baumbach, P.-C. Ho, T. A. Sayles, M. B. Maple, R. Wawryk, T. Cichorek, A. Pietraszko, and Z. Henkie, The filled skutterudite $\mathrm{CeOs}_{4} \mathrm{As}_{12}$ : A hybridization gap semiconductor, Proc. Natl. Acad. Sci. USA 105, 17307 (2008).

[18] G. P. Meisner, Superconductivity and magnetic order in ternary rare earth transition metal phosphides, Phys. B (Amsterdam, Neth.) 108, 763 (1981).

[19] E. D. Bauer, N. A. Frederick, P.-C. Ho, V. S. Zapf, and M. B. Maple, Superconductivity and heavy fermion behavior in $\mathrm{PrOs}_{4} \mathrm{Sb}_{12}$, Phys. Rev. B 65, 100506(R) (2002).

[20] I. Shirotani, S. Sato, C. Sekine, K. Takeda, I. Inagawa, and T. Yagi, Superconductivity of the new skutterudite compound $\mathrm{La}_{x} \mathrm{Rh}_{4} \mathrm{P}_{12}$ prepared at high pressure, J. Phys.: Condens. Matter 17, 7353 (2005).

[21] T. A. Sayles, R. E. Baumbach, W. M. Yuhasz, M. B. Maple, L. Bochenek, R. Wawryk, T. Cichorek, A. Pietraszko, Z. Henkie, and P.-C. Ho, Superconductivity and crystalline electric field effects in the filled skutterudite $\mathrm{PrRu}_{4} \mathrm{As}_{12}$, Phys. Rev. B 82, 104513 (2010).

[22] N. Takeda and M. Ishikawa, The ferromagnetic Kondo-lattice compound $\mathrm{SmFe}_{4} \mathrm{P}_{12}$, J. Phys.: Condens. Matter 15, L229 (2003).

[23] T. A. Sayles, W. M. Yuhasz, J. Paglione, T. Yanagisawa, J. R. Jeffries, M. B. Maple, Z. Henkie, A. Pietraszko, T. Cichorek, R. Wawryk, Y. Nemoto, and T. Goto, Thermodynamic and 
transport studies of the ferromagnetic filled skutterudite compound $\mathrm{PrFe}_{4} \mathrm{As}_{12}$, Phys. Rev. B 77, 144432 (2008).

[24] R. Higashinaka, K. Takeda, T. Namiki, Y. Aoki, and H. Sato, Anomalous low-lying thermal excitations deep inside the ferromagnetic state in filled skutterudite $\mathrm{NdFe}_{4} \mathrm{As}_{12}$, J. Phys. Soc. Jpn. 82, 114710 (2013).

[25] T. Cichorek, A. Rudenko, P. Wiśniewski, R. Wawryk, L. Kepiński, A. Pietraszko, and Z. Henkie, Schottky-like anomaly on the border of localized ferromagnetism in the filled skutterudite $\mathrm{NdOs}_{4} \mathrm{As}_{12}$, Phys. Rev. B 90, 195123 (2014).

[26] K. Iwasa, Y. Watanabe, K. Kuwahara, M. Kohgi, H. Sugawara, T. D. Matsuda, Y. Aoki, and H. Sato, Crystal-structure modulation in the anomalous low-temperature phase of $\mathrm{PrFe}_{4} \mathrm{P}_{12}$, Phys. B (Amsterdam, Neth.) 312-313, 834 (2002).

[27] M. B. Maple, N. P. Butch, N. A. Frederick, P.-C. Ho, J. R. Jeffries, T. A. Sayles, T. Yanagisawa, W. M. Yuhasz, S. Chi, H. J. Kang, J. W. Lynn, Pengcheng Dai, S. K. McCall, M. W. McElfresh, M. J. Fluss, Z. Henkie, and A. Pietraszko, Fielddependent ordered phases and Kondo phenomena in the filled skutterudite compound $\mathrm{PrOs}_{4} \mathrm{As}_{12}$, Proc. Natl. Acad. Sci. USA 103, 6783 (2006).

[28] I. Shirotani, T. Uchiumi, K. Ohno, C. Sekine, Y. Nakazawa, K. Kanoda, S. Todo, and T. Yagi, Superconductivity of filled skutterudites $\mathrm{LaRu}_{4} \mathrm{As}_{12}$ and $\mathrm{PrRu}_{4} \mathrm{As}_{12}$, Phys. Rev. B 56, 7866 (1997).

[29] L. Bochenek, R. Wawryk, Z. Henkie, and T. Cichorek, Evidence for multiple superconducting gaps in the filled skutterudite compound $\mathrm{LaRu}_{4} \mathrm{As}_{12}$, Phys. Rev. B 86, 060511(R) (2012).

[30] K. Matsuhira, C. Sekine, M. Wakeshima, Y. Hinatsu, T. Namiki, K. Takeda, I. Shirotani, H. Sugawara, D. Kikuchi, and H. Sato, Systematic study of lattice specific heat of filled skutterudites, J. Phys. Soc. Jpn. 78, 124601 (2009).

[31] S. Ram, V. Kanchana, and M. C. Valsakumar, Skutterudites under pressure: An ab initio study, J. Appl. Phys. 115, 093903 (2014).

[32] H. M. Tütüncü, E. Karaca, and G. P. Srivastavva, Electronphonon superconductivity in the filled skutterudites $\mathrm{LaRu}_{4} \mathrm{P}_{12}$, $\mathrm{LaRu}_{4} \mathrm{As}_{12}$, and $\mathrm{LaPt}_{4} \mathrm{Ge}_{12}$, Phys. Rev. B 95, 214514 (2017).

[33] J. Klotz, K. Götze, V. Lorenz, Y. Prots, H. Rosner, H. Harima, L. Bochenek, Z. Henkie, T. Cichorek, I. Sheikin, and J. Wosnitza, Fermi surface investigation of the filled skutterudite $\mathrm{LaRu}_{4} \mathrm{As}_{12}$, Phys. Rev. B 100, 205106 (2019).

[34] Y. Mizukami, M. Konczykowski, Y. Kawamoto, S. Kurata, S. Kasahara, K. Hashimoto, V. Mishra, A. Kreisel, Y. Wang, P. J. Hirschfeld, Y. Matsuda, and T. Shibauchi, Disorder-induced topological change of the superconducting gap structure in iron pnictides, Nat. Commun. 5, 5657 (2014).

[35] T. Takenaka, Y. Mizukami, J. A. Wilcox, M. Konczykowski, S. Seiro, C. Geibel, Y. Tokiwa, Y. Kasahara, C. Putzke, Y. Matsuda, A. Carrington, and T. Shibauchi, Full-Gap Superconductivity Robust Against Disorder in Heavy-Fermion $\mathrm{CeCu}_{2} \mathrm{Si}_{2}$, Phys. Rev. Lett. 119, 077001 (2017).

[36] T. Dahm and K. Ueda, NMR Relaxation and Resistivity from Rattling Phonons in Pyrochlore Superconductors, Phys. Rev. Lett. 99, 187003 (2007).

[37] V. Keppens, D. Mandrus, B. C. Sales, B. C. Chakoumakos, P. Dai, R. Coldea, M. B. Maple, D. A. Gajewski, E. J. Freeman, and S. Bennington, Localized vibrational modes in metallic solids, Nature (London) 395, 876 (1998).

[38] T. Yanagisawa, H. Saitoh, H. Hidaka, H. Amitsuka, T. Cichorek, Z. Henkie, and M. B. Maple, Elastic constants of filled skutterudite $\mathrm{LaRu}_{4} \mathrm{As}_{12}$, J. Phys.: Conf. Ser. 273, 012065 (2011).

[39] M. M. Koza, D. Adroja, N. Takeda, Z. Henkie, and T. Cichorek, Vibrational dynamics of filled skutterudites $\mathrm{La}_{4} X_{12}(T=\mathrm{Fe}$, $\mathrm{Ru}, \mathrm{Os}, X=\mathrm{As}, \mathrm{Sb})$, J. Phys. Soc. Jpn. 82, 114607 (2013).

[40] M. Shimizu, H. Amanuma, K. Hachitani, H. Fukazawa, Y. Kohori, T. Namiki, C. Sekine, and I. Shirotani, ${ }^{75}$ As-NQR studies of superconducting filled skutterudites $\operatorname{PrRu}_{4} \mathrm{As}_{12}$ and $\mathrm{LaRu}_{4} \mathrm{As}_{12}$, J. Phys. Soc. Jpn. 77, 104705 (2008).

[41] J. Juraszek, R. Wawryk, Z. Henkie, M. Konczykowski, and T. Cichorek, Symmetry of Order Parameters in Multiband Superconductors $\mathrm{LaRu}_{4} \mathrm{As}_{12}$ and $\mathrm{PrOs}_{4} \mathrm{Sb}_{12}$ Probed by Local Magnetization Measurements, Phys. Rev. Lett. 124, 027001 (2020).

[42] M. Putti, M. Affronte, C. Ferdeghini, P. Manfrinetti, C. Tarantini, and E. Lehmann, Observation of the Crossover from Two-Gap to Single-Gap Superconductivity Through Specific Heat Measurements in Neutron-Irradiated $\mathrm{MgB}_{2}$, Phys. Rev. Lett. 96, 077003 (2006).

[43] K. Oshiba and T. Hotta, Strong-coupling theory of rattlinginduced superconductivity, J. Phys. Soc. Jpn. 80, 094712 (2011).

[44] T. Isono, D. Iguchi, T. Matsubara, Y. Machida, B. Salce, J. Flouquet, H. Ogusu, J. Yamaura, Z. Hiroi, and K. Izawa, Understanding of the temperature-pressure phase diagram of $\beta$-pyrochlore oxides: A role of anhamonicity on superconductivity, J. Phys. Soc. Jpn. 82, 114708 (2013).

[45] J. Chang, I. Eremin, and P. Thalmeier, Cooper-pair formation by anharmonic rattling modes in the $\beta$-pyrochlore superconductor $\mathrm{KOs}_{2} \mathrm{O}_{6}$, New J. Phys. 11, 055068 (2009). 